\title{
El coeficiente de eficacia del humo de sílice
}

\author{
Mª PILAR ALAEJOS GUTIÉRREZ. Dr.Ingeniero de Caminos, Canales y Puertos. \\ Lab.Central de Estructuras y Materiales del CEDEX \\ MANUEL FERNÁNDEZCÁNOVAS.Dr.Ingeniero deConstrucción.Catedrático \\ de Materiales de la ETSI de CC y P de la UPM
}

Fecha de recepción: 9-III-98

Fecha de aceptación: 27-V-98

ESPAÑA

\begin{abstract}
RESUMEN
El humo de sílice es una adición de carácter puzolánico que se utiliza fundamentalmente para conseguir hormigones de alta resistencia. La normativa española actual de adiciones exige que el humo de sílice cumpla unos requisitos determinados para poder utilizarse como adición al hormigón. Cumpliendo estos requisitos, pueden utilizarse microsílices de características distintas que influyen de distinta manera en las propiedades del hormigón. La ponencia expone los resultados experimentales obtenidos en el Laboratorio Central de Estructuras y Materiales del CEDEX con tres microsílices de diferente procedencia. Los resultados han permitido determinar que el coeficiente de eficacia del humo de silice puede tener valores muy diferentes, incluso con adiciones que cumplan la normativa vigent. Se compara el valor de este coeficiente de eficacia con el incluido en el texto de la futura EHE.
\end{abstract}

\section{SUMMARY}

Silica fume is a pozzolanic addition specially used to get high resistance concrete. Present Spanish standards on addidites dictate that silica fumes have to comply with specific requirements when used as concrete addition. Once it has been proved they comply with those requirements, microsilicas of different characteristics can be used, which influence concrete properties in different ways. This paper discloses the experimental results obtained in the Central Laboratory of Structures and Materials of the CEDEX with three microsilicas from different sources. Through the results it has been observed that the coefficient of efficiency of silica fume can have very different values, although complying with the standards in force. The value of this coefficient of efficiency is compared to the one included in text of the future EHE.

\section{INTRODUCCIÓN}

El humo de sílice ha irrumpido en poco tiempo en el mundo de la tecnología del hormigón. La próxima Instrucción EHE lo incluirá como adición puzolánica no sólo para hormigón en masa y armado sino también para hormigón pretensado, campo vedado hasta el momento para las cenizas volantes.

El hecho de que el humo de sílice sea un componente indispensable para la fabricación de hormigones de muy alta resistencia hace que esta adición se considere en general como un componente que mejora la calidad de cualquier hormigón que lo incorpore en su dosificación. Esta consideración de carácter general debe aplicarse con precaución a cada caso particular, ya que los efectos beneficiosos del humo de sílice en la resistencia y durabilidad del hormigón van acompañados de molestos efectos secundarios, como su efecto en la consistencia del hormigón, que es necesario conocer, controlar y minimizar.

El gran éxito del humo de sílice radica en su notable efectividad química, si se compara con la obtenida con las cenizas. Esta efectividad es consecuencia de dos propiedades importantes:

a) $\mathrm{Su}$ elevado contenido de compuestos químicamente activos, fundamentalmente sílice amorfa, que constituye normalmente alrededor del $90 \%$ de su composición total.

b) Su gran finura, que la hace muy reactiva.

Por ambos motivos esta adición logra unas mejoras notables en la resistencia y durabilidad del hormigón utilizada en muy pequeñas proporciones: del 5 al $15 \%$ del peso de cemento. 
El permitir la utilización de una adición determinada como componente del hormigón, exige establecer unos requisitos mínimos en sus características que garanticen que el hormigón alcanzará las propiedades esperadas. En el caso del humo de sílice, la Instrucción EHE exigirá los siguientes requisitos:

- $\mathrm{SiO}_{2}$, según UNE EN 196-2>85\%

- $\mathrm{Cl}^{-}$, según UNE EN 196-2<0,10\%

- Pérdida al fuego, según UNE EN 196-2 <5\%

- Índice de actividad resistente, según UNE EN 196-1

$>100 \%$

Asimismo, se prescribe que no se podrá añadir humo de sílice al hormigón en cantidad superior al $10 \%$ del peso de cemento, y que sólo se utilizará la adición con cementos CEM I según la Instrucción RC-97. Cuando se fabrica un hormigón con adición, la EHE permite determinar el contenido de cemento para comprobar si se cumplen los requisitos de durabilidad según la fórmula $\mathrm{C}+\mathrm{KF}$, siendo $\mathrm{C}$ el contenido de cemento, $\mathrm{F}$ el contenido de adición y $\mathrm{K}$ el coeficiente de eficacia de la adición. El coeficiente de eficacia indica la ayuda que proporciona el humo de sílice al hormigón, en comparación con el cemento para resistir ambientes agresivos. La EHE asigna un coeficiente de eficacia de 2 al humo de sílice y lo reduce a 1 si la relación agua/cemento del hormigón es superior a 0,45 en ambientes de helada.

El presente artículo indica el valor del coeficiente de eficacia obtenido en términos de resistencia del hormigón del humo de sílice, y se compara con el valor recogido en la EHE.

\section{COEFICIENTE DE EFICACIA DEL HUMODE SÍLICE}

En el plan de ensayos se dosificaron hormigones con distintos porcentajes de humo de sílice $(0,10,15 \%$ en peso de cemento) y dos cementos diferentes: un I45/A y un I55/A (clasificación según Instrucción RC-93). La relación a/c varió de 0,26 a 0,40 para las mezclas con adición y de 0,26 a 0,65 sin ella. Se llenaron cilindros de $15 \times 30 \mathrm{~cm}$ y se conservaron en cámara húmeda hasta los 28 días de edad, momento de su rotura.

El método de determinar el coeficiente de eficacia partió de fijar una curva de resistencias para el hormigón del tipo $\mathrm{f}_{\mathrm{cm}}=\mathrm{e}^{\mathrm{A}-\mathrm{p} a / \mathrm{c}}$. " $\mathrm{A}$ " depende únicamente del árido utilizado en el hormigón y " $p$ " del material conglomerante. Con los datos experimentales se ajustaron las correspondientes curvas de regresión para los dos cementos y distintos porcentajes de humo de sílice.

Teniendo en cuenta que el tipo de árido utilizado en las dosificaciones fue el mismo, el término "A" debe salir sensiblemente similar en las regresiones encontradas, y así fue. Se adoptó un valor único de "A" determinado como media de los seis valores obtenidos en las curvas de regresión.

Fijando el valor de "A" se calcula el de "p" para cada una de las regresiones, obteniendo así lo que hemos denominado "curvas propuestas". Las curvas experimental y propuesta coinciden sensiblemente, como se muestra en la Figura1. para el cemento I45A. Los valores de "p" para cada una de las curvas propuestas se recogen en la Tabla 1. El valor de "A" es 7,27 para todas ellas.

Con estas curvas es inmediato determinar el valor del coeficiente de eficacia: suponiendo una curva general de resistencias: $f_{c m}=e^{A-p_{0}}$ a/c(1+rK) , entonces $K=1 / r\left(p_{0} / p-1\right)$ Siendo $\mathrm{K}$ el coeficiente de eficacia, $\mathrm{r}=\mathrm{el}$ contenido de humo de sílice en peso de cemento, $\mathrm{p}_{0}$ el valor de la Tabla 1 para $r=0 \%$ y p el valor para $r=10$ ó $15 \%$.

Los valores del coeficiente de eficacia así obtenido se recogen en la Tabla 2 , representados gráficamente en la Figura 2.

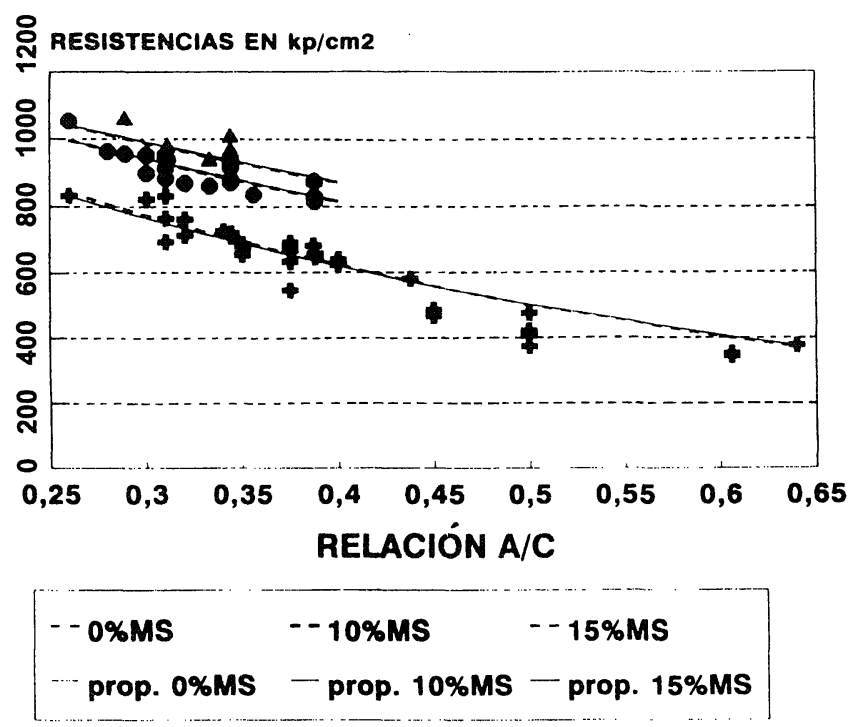

Figura 1.- Relación entre regresiones y curvas propuestas.

TABLA 1

Valor de "p" en la curva propuesta

\begin{tabular}{|c|c|c|}
\hline & $\mathbf{I 4 5 A}$ & $\mathbf{I 5 5 A}$ \\
\hline $\mathbf{M S}$ & $\mathbf{p}$ & $\mathbf{p}$ \\
\hline \hline $\mathbf{0} \%$ & 2,10 & 1,97 \\
$\mathbf{1 0 \%}$ & 1,41 & 1,31 \\
$\mathbf{1 5 \%}$ & 1,24 & 1,15 \\
\hline
\end{tabular}


TABLA 2

Valor del coeficiente de eficacia

\begin{tabular}{|c|c|c|}
\hline & CEMENTO I45A & CEMENTO I55A \\
\hline MS & K & K \\
\hline $10 \%$ & 4,89 & 5,03 \\
$15 \%$ & 4,62 & 4,75 \\
\hline
\end{tabular}

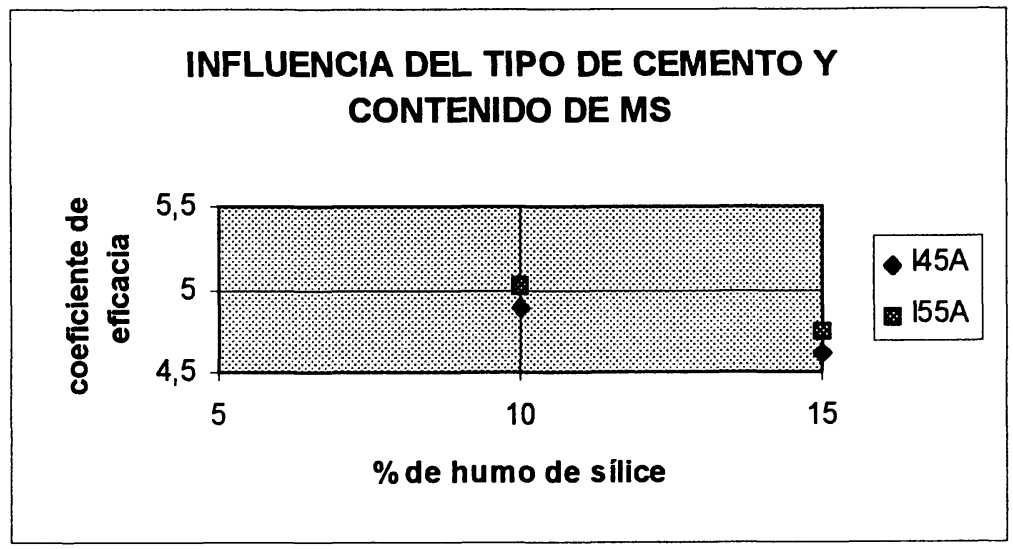

Figura 2.- Coeficiente de eficacia del cemento I45A y I55A con 10 y $15 \%$ de humo de silice.

\section{INFLUENCIA DEL TIPO DE CEMENTO}

La Figura 2 refleja un leve incremento del coeficiente de eficacia utilizando un cemento I55/A. El incremento es muy pequeño, $y$ hay que considerar la negativa influencia que sobre la consistencia tiene el utilizar un cemento I55/A, en general de mayor finura que los de menor categoría resistente.

Hay que tener en cuenta que todos los datos con humo de sílice de que se dispone corresponden a hormigones con adición de relación a/c menor de 0,40 . El contenido de agua de estos hormigones es muy bajo, por lo que llega un momento en que no existe agua disponible para que las reacciones químicas de hidratación y puzolánicas continúen. Además, al tratarse de hormigones de muy baja porosidad, el curado resulta menos efectivo, ya que el agua que se aporta no accede fácilmente al interior. Por estas razones, mucho cemento queda sin hidratar por lo que los cementos I55/A no desarrollan todo el potencial químico (correspondiente a su finura y composición) por falta de agua, lo cual se refleja en pequeños incrementos de resistencia. Es probable que la diferencia entre ambos cementos se acentúe con relaciones agua/cemento mayores. También es probable que el cemento I55/A, gracias a su mayor finura reaccione más rápidamente y la diferencia de resistencia con un cemento I45/A sea más acusada a cortas edades que a 28 días, edad que tienen los hormigones comparados en esta fase.

\section{INFLUENCIA DEL PORCENTAJE DE HUMO DE SÍLICE}

Los datos recogidos en la Tabla 2 indican que los coeficientes de eficacia en las mezclas con $10 \%$ de humo de sílice son mayores que en las mezclas con $15 \%$.

Este comportamiento responde también al hecho de que se trata de hormigones de muy baja relación agua/cemento, por lo que la cantidad de agua es insuficiente para completar las reacciones puzolánicas. Las diferencias obtenidas con gran probabilidad serán mayores en hormigones de mayor relación agua/cemento.

Por otra parte, y en cuanto a la influencia del porcentaje de humo de sílice en la resistencia final del hormigón, no hay que perder de vista que el volumen de portlandita liberada en la hidratación del cemento es fijo y depende de su composición química y agua disponible para las reacciones. El humo de sílice es una adición muy activa, que con una cantidad pequeña es capaz de consumir toda la portlandita generada por el cemento. Esta cantidad depende de la composición química del cemento y de la pureza del humo de sílice, y siempre en la hipótesis de que existe suficiente agua para que el cemento se hidrate en su totalidad y se completen las reacciones puzolánicas. Cantidades por encima del contenido de adición que agota toda la portlandita no reaccionan químicamente, influyendo únicamente en la resistencia por un efecto de 
relleno de los microporos del hormigón, lo cual supone un incremento de resistencia inferior al esperado.

Este comportamiento justifica el hecho de limitar el contenido de humo de sílice en el hormigón: Los incrementos de resistencia conseguidos son pequeños, aumentando en exceso la adición y, a cambio, se perjudica notablemente la consistencia del hormigón.

\section{INFLUENCIA DE LA RELACIÓN AGUA/CEMENTO}

La Figura 3 recoge la relación entre resistencia del hormigón con humo de sílice (para 5,10 y 15\%) y hormigón patrón para distintas relaciones agua/cemento. La extrapolación para a/c superior a 0,40 no está contrastada experimentalmente, por lo que hay que considerarla con precaución. La comparación en resistencias se refiere a mezclas con adición de un 5, 10 y $15 \%$ de humo de sílice, no de reemplazamiento. La figura muestra que el incremento de resistencia que proporciona el humo de sílice es mayor cuando se aumenta la relación a/c. Esto está de acuerdo con la mayor disponibilidad de agua que pueda aprovechar al máximo el efecto puzolánico del humo de sílice mencionado anteriormente.

Estequiométricamente se demuestra que en el caso hipotético de completarse la hidratación, un cemento de tipo medio $\left(\mathrm{SC}_{3}=50 \%\right)$ genera portlandita suficiente para reaccionar con un $15 \%$ de humo de sílice de pureza $85 \%$, por lo que cantidades superiores al $15 \%$ no aportan actividad puzolánica alguna y tan sólo pueden tener efecto de relleno. El 15\% de humo de sílice, sin embargo, supone una actividad puzolánica adicional al $10 \%$, que proporciona incrementos de resistencia no despreciables al menos en hormigones de relación agua/cemento normal, a la vista de la Figura 3. El límite del $10 \%$ de la Instrucción podría, por tanto, ser reconsiderado y elevado al $15 \%$.

\section{RELACION IA.R Y COETICIENTE DE EFI- CACIA}

Uno de los requisitos que exigirá la EHE al humo de sílice es que cumpla un índice de actividad resistente mínimo del $100 \%$, ensayo realizado en mortero sustituyendo un $10 \%$ de cemento por humo de sílice.

En una publicación anterior ${ }^{(1)}$ se estableció la relación que, por concepto, debiera existir entre el resultado de este ensayo de caracterización de la adición y el coeficiente de eficacia que posee. En aquella publicación se determinó que las cenizas deberían cumplir un I.A.R. mínimo de 0,80 , para obtener un coeficiente de eficacia mínimo de 0,40 .

Utilizando los datos experimentales de resistencia con humo de sílice obtenidos a 7 y 28 días de edad, se determinó el I.A.R. teórico de la adición para ambas edades. Para ello, y partiendo de los datos experimentales, se obtuvieron las curvas propuestas según se ha descrito anteriormente, correspondientes a las fórmulas

$f_{c m 7 d}=e^{7,27-2,60(a / c(1+2,26 r))}$ y $f_{c m 28 d}=e^{7,27-2,10(a / c(1+4,89 r))}$

Como se observa en las curvas 2,26 y 4,89 son los coeficientes de eficacia experimentalmente obtenidos para un contenido de humo de sílice del $10 \%$ en peso de cemento $(r=0,1)$, a los 7 y 28 días de edad respectivamente.

El procedimiento seguido para determinar el I.A.R. a partir de estos coeficientes de eficacia parte de las siguientes hipótesis:

-La resistencia del mortero sigue la misma ley que la del hormigón fabricado con el mismo cemento y el mismo árido. Esta hipótesis es la consecuencia de considerar el mortero como un hormigón con árido de tamaño máximo $5 \mathrm{~mm}$.

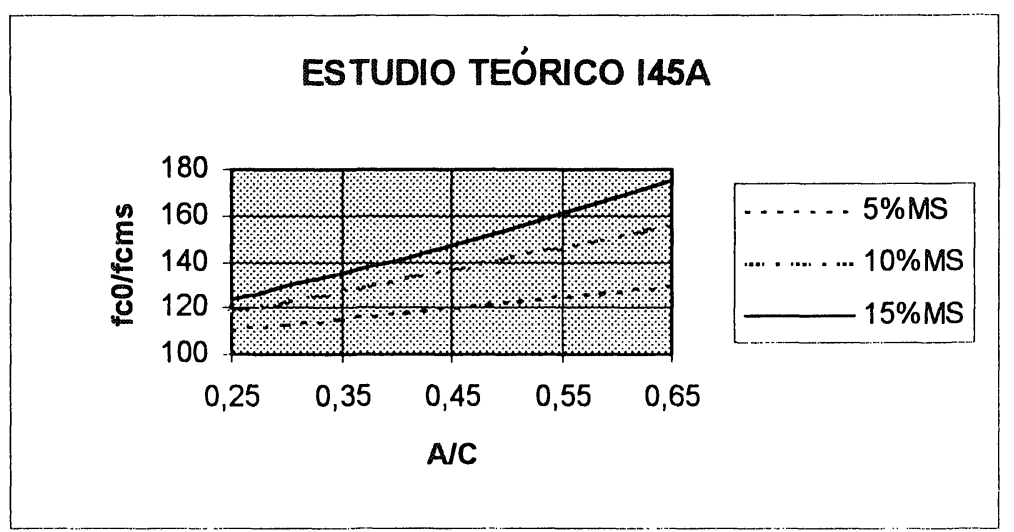

Figura 3.- Influencia de la relación agua/cemento, en la resistencia del hormigón con humo de sílice. 
-El valor de la constante " $A$ " de la curva de resistencia es el único parámetro que contempla la influencia del árido sobre la resistencia del mortero. Como el I.A.R. es la relación entre mortero patrón y mortero con adición, es independiente del árido con el que se ha fabricado dicho mortero.

El ensayo del I.A.R. se realiza con un mortero patrón de relación $\mathrm{a} / \mathrm{c}=0,5$ y un mortero con el mismo contenido de agua, sustituyendo un $10 \%$ de cemento por humo de sílice. Por tanto, para el mortero patrón $\mathrm{r}=0, \mathrm{a} / \mathrm{c}=0,5 \mathrm{y}$ para el mortero de humo de sílice $r=0,1 / 0,9$ y a/c $=0,55$. Con estos datos introducidos en cada una de las curvas anteriores se obtienen dos valores cuyo cociente es el I.A.R teórico. Este dato se comparó con el obtenido experimentalmente en el ensayo correspondiente. Los resultados se recogen en la Tabla 3. Dentro de lo limitado de los datos, se observa una perfecta coincidencia entre los valores calculados y los obtenidos experimentalmente

La correlación establecida entre I.A.R. del mortero y coeficiente de eficacia permite determinar el valor de éste partiendo del I.A.R. experimental de la adición considerada, con las siguientes ventajas:

-El ensayo del I.A.R. es requisito indispensable para la utilización de una adición puzolánica en el hormigón, por lo que es un dato siempre controlado y conocido.
-El permitir conocer el coeficiente de eficacia de una adición en el hormigón a partir de un ensayo realizado sobre mortero tiene las ventajas de la comodidad (fabricar mortero en vez de hormigón) y la precisión (el ensayo de resistencias en mortero presenta menos dispersión).

\section{INFLUENCIA DE LA EDAD Y EL TIPO DE HUMO DE SÍLICE}

Se realizaron ensayos sobre mortero para determinar la influencia de la edad y el tipo de humo de sílice en el I.A.R. La correlación de estos resultados con el coeficiente de eficacia antes establecida permitió predecir la evolución del valor del coeficiente de eficacia en el tiempo y la influencia del humo de sílice empleado.

Se utilizaron tres humos de sílice diferentes identificados como 1, 2 y 3 . Se fabricaron morteros patrón sin humo de sílice y morteros con sustitución del $10 \%$ de cemento por humo de sílice para romper a 2, 7, 28 y 90 días de edad. Los valores del I.A.R. se recogen en la Tabla 4. Con estos datos se calcularon los coeficientes de eficacia de la adición en un hormigón con el $10 \%$ en peso de cemento de humo de sílice y fabricado con el mismo cemento utilizado en los morteros. Los resultados se recogen en la Tabla 5. La representación gráfica de la evolución del coeficiente de eficacia se muestra en la

TABLA 3

Comparación entre el I. A. R. y calculado.

\begin{tabular}{|c|c|c|c|}
\hline Edad & K & I.A.R. calculado & I.A.R. experimental \\
\hline 7 dias & 2,26 & $116 \%$ & $117 \%$ \\
\hline 28 días & 4,89 & $134 \%$ & $135 \%$ \\
\hline
\end{tabular}

TABLA 4

Influencia de la edad y el humo de sílice en el I. A. R.

\begin{tabular}{|c|c|c|c|c|}
\hline \multirow{2}{*}{$\begin{array}{c}\text { Humo de } \\
\text { sílice }\end{array}$} & \multicolumn{4}{|c|}{ I.A.R. experimental } \\
\cline { 2 - 5 } & $2 \mathrm{~d}$ & $7 \mathrm{~d}$ & $28 \mathrm{~d}$ & $90 \mathrm{~d}$ \\
\hline 1 & 107 & 117 & 134 & 123 \\
\hline 2 & 115 & 118 & 134 & 128 \\
\hline 3 & 106 & 107 & 120 & 117 \\
\hline
\end{tabular}


TABLA 5

Influencia de la edad y el humo de sílice en el coeficiente de eficacia teórico

\begin{tabular}{|c|c|c|c|c|}
\hline \multirow{2}{*}{$\begin{array}{c}\text { Humo de } \\
\text { sílice }\end{array}$} & \multicolumn{4}{|c|}{ Coeficiente de eficacia teórico } \\
\cline { 2 - 5 } & $2 \mathrm{~d}$ & $7 \mathrm{~d}$ & $28 \mathrm{~d}$ & $90 \mathrm{~d}$ \\
\hline $\mathbf{1}$ & 1,35 & 2,37 & 4,86 & 3,97 \\
\hline $\mathbf{2}$ & 1,80 & 2,44 & 4,84 & 4,80 \\
\hline $\mathbf{3}$ & 1,34 & 1,56 & 3,09 & 3,06 \\
\hline
\end{tabular}

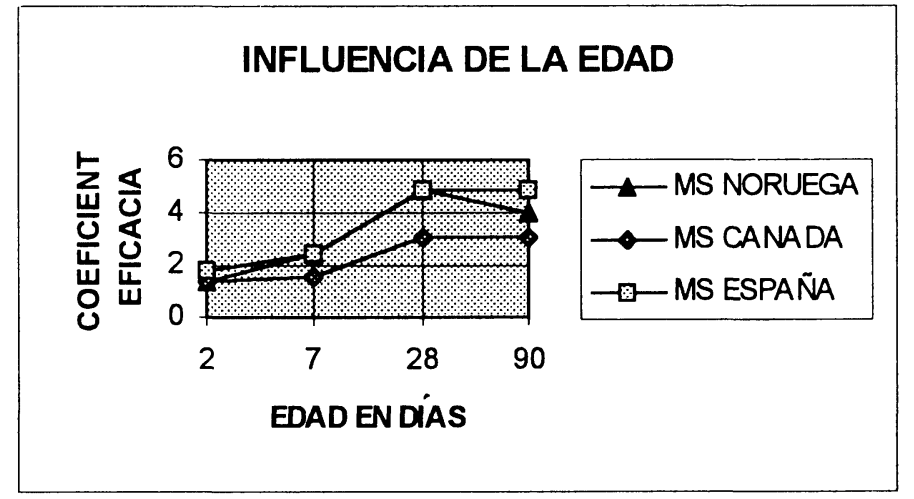

Figura 4.- Representación gráfica de la influencia de la edad y el tipo de humo de sílice.

Figura 4. A la vista de estos resultados, cabe hacer las siguientes consideraciones:

-La actividad puzolánica de los tres humos de sílice estudiados es efectiva a los 2 días de edad, y su actividad es tanto mayor cuanto mayor es la edad, hasta los 28 días de edad.

-A los 90 días de edad se ha paralizado prácticamente la actividad puzolánica, como indica que hecho de que el coeficiente de eficacia es igual o inferior al obtenido a los 28 días de edad.

-Existen notables diferencias entre los coeficientes de eficacia conseguidos entre unos y otros humos de sílice, consecuencia de las diferentes composiciones químicas $\mathrm{y}$ finuras que presentan.

-El valor más bajo obtenido del coeficiente de eficacia a los 28 días de edad fue de 3 aproximadamente, y corresponde al humo de sílice marcado como 3. La composición química indicó que el contenido de sílice total de este humo de sílice era de $87 \%$, casi al límite del mínimo exigido por la EHE.

\section{REPERCUSIÓN EN LA NORMATIVA}

La futura Instrucción EHE exigirá al humo de sílice un I.A.R. mínimo a los 28 días de edad del $100 \%$, permitiendo un contenido máximo del $10 \%$ en peso de cemento. Además, para garantizar un hormigón de adecuada durabilidad exige unos contenidos mínimos de cemento calculados, en el caso de hormigón con humo de sílice con coeficiente de eficacia de 2 ( 1 en el caso de a/c mayor de 0,45 y ambientes con helada o utilización de sales fundentes).

Con los resultados experimentales obtenidos (insistiendo en que la comparación es de coeficientes de eficacia en términos de resistencia a compresión y en términos de durabilidad) se pueden hacer las siguientes consideraciones:

-El procedimiento expuesto que relaciona el I.A.R. y el coeficiente de eficacia permite deducir que un humo de sílice con I.A.R. del $100 \%$ sólo garantiza un coeficiente de eficacia de 1, muy por debajo del 2 asignado en el capítulo de durabilidad. 
-Si el coeficiente de eficacia que en condiciones normales se va a atribuir al humo de sílice es de 2 , ello implica exigir que presente un I.A.R. mínimo del $110 \%$ a los 28 días de edad.

-Teniendo en cuenta que un humo de sílice con el mínimo contenido de sílice permitido, proporciona un I.A.R. a los 28 días del $120 \%$ y un coeficiente de eficacia de 3 , cabe preguntarse si es realista asignar un coeficiente de eficacia de 2 a esta adición. Esta asignación resulta aún más incierta si se consulta alguna literatura al respecto ${ }^{(2),(3)}$, donde, en general, el humo de sílice tiene un coeficiente de eficacia a los 28 días superior a 3 , en condiciones de curado normales.

-Para que se pueda deducir con mayor precisión el coeficiente de eficacia a partir del I.A.R. es conveniente que el ensayo en mortero contenga adición en el mismo porcentaje que el máximo permitido en el hormigón. Esto implica que, en el caso del humo de sílice, el mortero debería llevar un $10 \%$ del humo de sílice en peso de cemento, en vez de en peso de material cementante total. Aunque, de este modo, las diferencias resultan pequeñas en ambos casos, y así se ha tenido en cuenta en el presente plan de ensayos, cuando se trata de adiciones que se permiten en mayor cuantía (cenizas volantes) estas diferencias aumentan.

\section{CONCLUSIONES}

-El humo de sílice resulta ser una adición puzolánica de elevada eficiencia para incrementar la resistencia del hormigón. El coeficiente de eficacia a los 28 días mínimo encontrado ha sido de 3 , para el caso de un humo de sílice que cumple estrictamente los requisitos exigidos por la EHE a esta adición.
-Se ha comprobado experimentalmente la buena correlación existente entre el I.A.R. y el coeficiente de eficacia, con las ventajas que roporta el poder determinar éste a partir del resultado del ensayo en mortero.

-El porcentaje en peso de cemento máximo admitido en el hormigón, según EHE del $10 \%$ podría ser reconsiderado y elevado al $15 \%$, a la vista de los resultados obtenidos. Haría falta una experimentación complementaria a la realizada con hormigones de relación agua cemento superior a 0,40 .

-El I.A.R. mínimo exigido por la EHE del 100\% resulta excesivamente bajo y poco conservador, si se asigna al humo de sílice un coeficiente de eficacia de 2. El valor debería elevarse, al menos, hasta un $110 \%$.

-Los resultados obtenidos indican que resulta más realista asignar un coeficiente de eficacia al humo de sílice de 3. En este caso el I.A.R. mínimo exigible sería del $120 \%$. Este extremo podría ser confirmado con una experimentación complementaria.

\section{BIBLIOGRAFÍA}

(1) P. ALAEJOS. "El coeficiente de eficacia de las cenizas volantes". Cemento-Hormigón, № 747, julio 1995, pp.746770 .

(2) M. MAAGE. "Efficiency Factors for Condensed Silica Fume in Concrete". Proceedings del Symposium on High Strength Concrete, Trondheim Norway 1989.

(3) K.G. BABU, P.V. SURYA PRAKASH. "Efficiency of Silica Fume in Concrete". Cement and Concrete Research, Vol.25, N16,pp.1273-1283. 